\title{
Polysèmes
}

Revue d'études intertextuelles et intermédiales

\section{Entre mythes helléniques et récits édifiants : hagiographies parodiques dans Under the Hill d'Aubrey Beardsley et Stories Toto Told Me de Frederick Rolfe}

Greco-Roman Myths and Edifying Stories: Parodic Hagiographies in Aubrey

Beardsley's Under the Hill and Frederick Rolfe's Stories Toto Told Me

Claire Masurel-Murray

\section{OpenEdition \\ Journals}

Édition électronique

URL : http://journals.openedition.org/polysemes/295

DOI : $10.4000 /$ polysemes.295

ISSN : 2496-4212

Éditeur

SAIT

\section{Référence électronique}

Claire Masurel-Murray, «Entre mythes helléniques et récits édifiants : hagiographies parodiques dans

Under the Hill d'Aubrey Beardsley et Stories Toto Told Me de Frederick Rolfe », Polysèmes [En ligne],

13 | 2015, mis en ligne le 13 octobre 2015, consulté le 19 avril 2019. URL : http://

journals.openedition.org/polysemes/295; DOI : 10.4000/polysemes.295

Ce document a été généré automatiquement le 19 avril 2019

Polysèmes 
Entre mythes helléniques et récits édifiants : hagiographies parodiques dans Under the Hill d'Aubrey Beardsley et Stories Toto Told Me de Frederick Rolfe

\author{
Greco-Roman Myths and Edifying Stories: Parodic Hagiographies in Aubrey \\ Beardsley's Under the Hill and Frederick Rolfe's Stories Toto Told Me
}

Claire Masurel-Murray

À la fin de l'ère victorienne, une véritable mode catholique se développe dans les milieux littéraires anglais. Certains auteurs décadents voient dans le catholicisme un réservoir de symboles et de mythes, mais aussi un patrimoine textuel aux multiples facettes, sans cesse réécrit, sans cesse recréé. Dans Marius the Epicurean, le roman de Walter Pater publié en 1885 , qui se déroule au début de l'ère chrétienne, Pater utilise l'image du palimpseste pour décrire les tombes chrétiennes construites à partir de stèles païennes plus anciennes, «marble taken, in some cases, from older pagan tombs-the inscription sometimes a palimpsest, the new epitaph being woven into the faded letters of an earlier one $»^{1}$. Cette image du palimpseste où se superposent inscriptions chrétiennes et inscriptions païennes est tout à fait apte à décrire des effets d'intertextualité qui jouent aussi bien sur la tradition gréco-latine que sur les codes chrétiens. L'intertextualité dans les œuvres de la Décadence prend en effet de multiples formes. Elle peut affleurer dans le texte lui-même sous forme de citations, d'allusions, de traductions. Elle peut aussi relever d'une stratégie générique. Le genre hagiographique, c'est-à-dire la vie de saint, devient à la fin du XIX siècle dans la littérature européenne l'objet d'une sorte de jeu littéraire, auquel se livrent notamment Gustave Flaubert, dans La Tentation de saint Antoine (1874) et «La Légende de saint Julien l'Hospitalier» (1877), Maurice Maeterlinck sur le mode parodique dans Le Miracle de saint Antoine (1920), et Oscar Wilde, chez qui les saints 
peuvent être des personnages de théâtre (l'ermite et la prostituée dans la courte pièce intitulée La Sainte Courtisane, saint Jean-Baptiste - Jonakaan - dans Salomé), ou les héros fictifs de contes tels que "The Happy Prince », «The Young King ", "The Fisherman and His Soul », où abondent les motifs hagiographiques («conversions », sacrifices, bonnes actions, miracles).

icle se penche sur ces stratifications littéraires dans les textes de deux écrivains fin de siècle: Aubrey Beardsley né en 1872, converti au catholicisme en 1897, et mort en 1898, plus connu pour ses illustrations mais aussi auteur d'un roman érotique, Under the Hill (1896), et Frederick Rolfe (1860-1913, converti en 1886), également connu sous le nom de "Baron Corvo " qu'il aimait se donner, qui fit paraître une série de contes catholicisants entre octobre 1895 et octobre 1896 dans The Yellow Book, revue quintessentielle du mouvement décadent. Ces contes furent rassemblés et publiés en 1898 sous le titre Stories Toto Told Me.

Ces deux œuvres proposent des réécritures parodiques de vies de saints, s'appropriant ainsi, dans des styles très différents, un exercice littéraire populaire à l'époque. Dans Under the Hill, Beardsley propose un résumé de la vie de sainte Rose de Lima qui pastiche une hagiographie publiée en Angleterre en 1847. Quant aux Stories Toto Told Me, ce sont des légendes « catholiques » racontées sur le mode mythologique. Un jeune Italien, Toto, $\mathrm{y}$ raconte des histoires où les saints, avatars des dieux de l'olympe, se disputent et se jouent des tours, batifolant entre la terre et l'au-delà. Rolfe propose sur un mode italianisant et paganisant une série d'hagiographies parodiques, évoquant tour à tour Sangiorgio, Santignazio of Loyola, Sanluigi, Sanvenanzio et Santagapito, Sangiuseppe, Sampietro et Sampaolo, et même « the mamma of Sampietro ».

On montrera comment Beardsley et Rolfe reprennent sur le mode de la parodie les codes de ce genre typiquement catholique qu'est le récit hagiographique, dont le caractère stéréotypé autorise le détournement, pour le vider de son sens religieux, en jouant à la fois sur la transparence et l'opacité: Beardsley offre ainsi au lecteur un véritable feuilletage de prétendus hypotextes, tout en occultant sa source véritable qu'il reprend pourtant quasiment mot pour mot, tandis que Rolfe dissimule sous des noms de saints des personnages directement tirés des Métamorphoses d'Ovide.

\section{La Tradition réinventée : influences catholiques et intertextes hagiographiques dans la littérature décadente}

Les auteurs de la fin de siècle puisent leur inspiration à des sources diverses. On trouve en particulier dans leurs œuvres un héritage littéraire essentiel, celui que représente le catholicisme. À ces artistes qui ne croient plus à la possibilité d'une parole originale, la tradition catholique offre un immense dépôt de textes qu'ils vont pouvoir se réapproprier. Les décadents anglais sont en effet des traditionalistes au sens où le passé est chez eux plus qu'une simple thématique : c'est un véritable mode d'écriture. Comme l'a démontré Linda Dowling dans Language and Decadence in the Victorian Fin de Siècle, le mouvement décadent surgit à la suite d'une grave crise linguistique qui remet en question le pouvoir du langage ${ }^{2}$. Face à la faillite d'une parole qui se fragmente jusqu'à la perte du sens, les décadents, habités par la conviction que la langue «vulgaire » est désormais incapable de dire le monde, vont puiser dans les œuvres et les formes 
poétiques du passé. Le catholicisme est perçu comme un patrimoine riche et magnifique, un refuge contre les effets dévastateurs d'une temporalité néfaste, qui ne peut s'exprimer que dans une artificialité consciente et pleinement assumée.

Loin d'être fondée sur la recherche de l'originalité et de l'unicité, l'écriture décadente, notamment chez Beardsley et Rolfe, repose sur une esthétique de la réécriture et de la répétition. Il s'agit de réécrire une religion enracinée dans son passé, non en créant une langue neuve, mais en réinventant la langue traditionnelle de l'Église, telle qu'elle se manifeste en particulier dans le récit hagiographique. L'intérêt des décadents pour les textes du passé trouve un écho dans la sacralisation, dans la doctrine catholique, de la longue succession de textes qui, depuis les temps apostoliques, forme la "Tradition ». Alors que le protestantisme affirme que l'Écriture est elle-même, sous l'influence de l'Esprit Saint, sa propre interprète (sola scriptura), le catholicisme reconnaît deux sources de connaissance de la Révélation, l'Écriture et la Tradition, dont l'Église est dépositaire. Le dépôt de la foi catholique n'est donc pas circonscrit à un texte sacré original, mais est composé d'une multitude de textes composés sur une période de deux mille ans, reproduisant en quelque sorte à l'infini une parole préexistante.

7 Cette idée fournit à Frederick Rolfe le prétexte de l'intrigue dans « About the Heresy of Fra Serafico", conte de la série des Stories Toto Told Me qui propose un commentaire ironique de cette notion catholique de tradition. Fra Serafico, jeune frère franciscain, connaît un grand succès dans sa prédication, jusqu'au jour où un jésuite jaloux, Padre Tonto Pappagallo (on voit que les noms des personnages ont une valeur à la fois ludique et programmatique) $)^{3}$, l'accuse d'hérésie sous prétexte qu'il prêche la doctrine suivante: "No one shall be crowned unless he has contended lawfully "4. Pour se défendre, le franciscain raconte alors un rêve qu'il a fait, dans lequel, mis en demeure de prouver son innocence devant Dieu, il explique au Très-Haut qu'il a emprunté cette phrase à la trenteseptième homélie de saint Grégoire le Grand sur le quatorzième chapitre de l'Évangile de Luc. Saint Grégoire, qui est alors appelé à la barre, explique que l'expression est tirée du cinquième verset du deuxième chapitre de la deuxième épître de saint Paul à Timothée. Saint Paul admet être l'auteur de la citation, mais souligne qu'il a écrit sous inspiration divine, et qu'il est donc impossible qu'il ait produit une hérésie ${ }^{5}$. Le verdict de Dieu (qui chez Rolfe parle toujours en lettres majuscules) donne raison au petit franciscain contre son adversaire jésuite :

THE WORDS WHICH YOU HAVE WRITTEN, SAMPAOLO, IN YOUR SECOND LETTER TO SANTIMOTEO, ARE NOT THE WORDS OF A MAN, BUT OF GOD HIMSELF; AND THE MATTER AMOUNTS TO THIS, THAT OUR LITTLE BROTHER HERE, WHO TOOK THE WORDS FROM SANGREGORIO, DETTO MAGNO, WHO TOOK THEM FROM YOU, WHO WERE DIVINELY INSPIRED TO WRITE THEM, HAS NOT BEEN GUILTY OF HERESY AT ALL, UNLESS GOD HIMSELF CAN ERR. ${ }^{6}$

On a donc ici une succession d'« auteurs » qui refusent d'assumer l'originalité de leurs propos, comme si la parole humaine ne pouvait être que citation et répétition, jusqu'à ce que l'on remonte à la source absolue, le verbe divin (le ton irrévérencieux du récit jette cependant le doute sur la validité de cette origine transcendante de la parole). La Tradition catholique est donc l'espace où résonne une parole reçue et transmise, plutôt que créée, aux échos démultipliés par une transmission séculaire. Elle fournit un immense réservoir intertextuel dans lequel les auteurs de la décadence ne vont cesser de puiser. 
9 En réécrivant les textes hagiographiques de la tradition catholique ou en en inventant d'autres, sur le mode de la parodie et du pastiche, Beardsley et Rolfe peuvent trouver leur voix propre tout en acceptant l'idée que l'écriture ne peut que véhiculer une langue morte ${ }^{7}$. Dans Stories Toto Told Me et Under the Hill, l'élément religieux est à la fois central et tenu à distance par le biais de l'humour, qui décrédibilise les faits rapportés, le contexte irrévérencieux désacralisant la parole et le geste religieux. Alors que le récit hagiographique a en principe une fonction propédeutique, car il est censé actualiser la présence divine dans la vie des saints et conduire à la foi, il n'est plus ici qu'un signe textuel parmi d'autres au sein d'une esthétique de l'hétéroclite.

Ces réécritures hagiographiques s'inscrivent dans un courant merveilleux qui fleurit dans les années 1890 , où l'imaginaire catholique joue souvent un rôle prépondérant, à l'opposé du courant réaliste et naturaliste. On peut citer les contes médiévalisants d'Arthur Christopher Benson (The Hill of Trouble, 1903), les contes "The Virgin of the Seven Daggers» (1889), «Pope Jacynth» (1904), mais aussi «St. Eudaemon and his OrangeTree» (1904) de Vernon Lee, et une myriade de textes d'auteurs peu connus publiés dans les revues décadentes telles que The Yellow Book, The Savoy ou The Hobby Horse. Ces contes catholicisants, qui ont souvent des saints pour protagonistes, se distinguent des récits fantastiques qui font florès à l'époque, car ils évoquent un monde où le surnaturel est la règle et se juxtapose sans heurt au monde réel, un univers fondamentalement harmonieux, même s'il connaît aussi la lutte du bien et du mal. Ils mettent en scène un espace où le prodige est souvent le vecteur d'un dénouement heureux, et non une agression menaçante qui viendrait déstabiliser la nature et déchirer la cohérence du monde réel comme dans le récit fantastique.

11 Ces textes se situent dans un territoire générique difficile à définir, entre l'hagiographie, qui prétend rapporter de manière fidèle les actions et les faits miraculeux mais véridiques de la vie d'un saint, la légende, qui se donne comme le récit d'événements qui se sont réellement produits et dont les acteurs sont connus, et qui en appelle - ou en a autrefois appelé - à la croyance, et le conte, qui possède un caractère de fiction avoué, ne requiert la crédulité du lecteur que pour le temps que dure le récit, et relève en fin de compte du pur divertissement. Ils appartiennent à la fois au merveilleux, qui implique, comme l'indique l'étymologie, un étonnement nuancé de crainte et d'admiration, et que Chateaubriand place au cœur de la poétique du christianisme ("Du Merveilleux », livre VI du Génie du christianisme), à la féerie, qui place d'emblée le surnaturel dans un univers fictif, et au mythe, qui offre une explication de l'inexplicable sous la forme d'un récit se rapportant à un état du monde antérieur à l'état présent. On a souvent l'impression qu'entre ces différentes formes de récit les décadents refusent de choisir. Ils voudraient croire, mais ne croient plus vraiment; ils prétendent ré-enchanter l'univers par le biais $\mathrm{du}$ merveilleux chrétien, mais leur vision $\mathrm{du}$ monde est fondamentalement désenchantée; ils sont fascinés par les phénomènes miraculeux, mais en même temps s'efforcent constamment de les placer à distance par différents moyens poétiques et narratifs. La réappropriation littéraire des mythes chrétiens et des légendes catholiques s'accompagne chez eux d'un mouvement de dé-crédibilisation et d'une disparition de la vocation édifiante du récit. L'embellissement qu'ils apportent à des faits devenus invérifiables semble signifier qu'ils n'y croient plus, l'esthétisation prenant le pas sur la foi. 


\section{Stories Toto Told Me : saints du paradis et dieux de l'Olympe}

12 Les contes catholicisants de la fin de siècle mettent souvent en scène une véritable mythologie, qui s'inspire autant des Métamorphoses d'Ovide que de la Légende dorée de Jacques de Voragine (ouvrage séminal du XIII ${ }^{\mathrm{e}}$ siècle racontant la vie d'environ centcinquante saints). Le culte des saints dans l'Église romaine a sans doute favorisé le développement de cette mythologie pagano-catholique, que dénonce la littérature pamphlétaire de l'époque. Pater abonde dans ce sens dans Marius the Epicurean lorsqu'il trace un parallèle entre la dévotion de Marc-Aurèle pour les idoles et le culte des saints : "If the comparison may be reverently made, there was something here of the method by which the catholic church has added the cultus of the saints to its worship of the one Divine Being $»^{8}$.

La lecture de la série des Stories Toto Told Me montre que les auteurs anticatholiques n'ont pas complètement tort lorsqu'ils dénoncent les affinités entre culte des saints, mythes grecs et latins, et idolâtrie. Les contes de Rolfe sont en effet des légendes hagiographiques racontées sur le mode mythologique, où les saints, avatars des dieux de l'olympe, sont appelés "gods». Ces récits souvent humoristiques, racontés sur un ton faussement naif, prêtent aux activités célestes de ces "dieux» catholiques des motivations humaines, comme dans la mythologie grecque et romaine. Le conteur puise la trame de ses récits dans un répertoire connu depuis longtemps, celui de la Légende dorée et du martyrologe romain, mais leur imprime sa marque propre en les paganisant, et en christianisant parallèlement certaines références grecques et romaines. Cora (plus connue sous le nom de Perséphone) devient Santacore, les saints jurent par Bacchus, Sansebastiano et Sampancrazio promènent dans les jardins du paradis leurs corps jeunes et nus de dieux grecs. "About Divinamore and the Maiden Anima» est une réécriture des aventures d'Éros et Psyché, et « Being an Epick of Sangiorgio, Protector of the Kingdom » transpose l'histoire de Persée dans un contexte catholique. Mais c'est dans «About a Vegetable Purgatory » que les références à la mythologie sont les plus nombreuses et les plus transparentes.

14 Le sujet de ce récit est le sort (relu par Rolfe) des âmes du purgatoire : « persons who have their purgatory in flowers, or in trees; persons who, for some little sort of sin, are pent in places from which they may not move, wearing unusual shapes which not their lovers, nor even their own mothers, recognize $»^{9}$. À partir de la question polémique du purgatoire, véritable pierre d'achoppement entre anglicans et catholiques ${ }^{10}$, Rolfe réécrit un certain nombre de mythes gréco-latins et d'épisodes des Métamorphoses.

15 Ainsi, Ovide relate l'histoire d'Attis dont la déesse Cybèle, qui l'aimait d'une passion chaste, avait fait le gardien de son temple à condition qu'il garde sa virginité. Cela devient chez Rolfe l'histoire d'Atiso qui promet de devenir le serviteur de la Vierge en se faisant prêtre. De même que, selon Ovide, Attis ne sut pas résister à l'amour de la nymphe Sagaritis, Atiso tombe amoureux d'une jeune fille nommée Sangarisa. Chez Ovide, Cybèle, folle de jalousie, abat l'arbre à la vie duquel celle de Sagaritis est liée et frappe de folie Attis qui s'émascule au cours d'une crise violente, et est ensuite de nouveau accueilli par la déesse qui le reprend à son service; chez Rolfe, la Vierge se contente de regarder Atiso avec des yeux si tristes qu'il est pris de remords, s'enfuit dans les bois où il mène une vie de prière et de pénitence, et devient prêtre. La peine qu'il reçoit au purgatoire est d'être 
transformé en pin : « a pine-tree, tall and slender, as he was at that time when he belied his promise, head veiled in sad-colour, drooping in regret $»^{11}$.

Quant à Chuporiso, qui dans le même conte tue un cerf appartenant à un couvent dédié à saint Michel archange (version rolfienne d'Apollon), et qui au purgatoire est changé en cyprès, son histoire rappelle étrangement celle de Cyparissos. Dans les Métamorphoses, ce jeune homme aimé d'Apollon tue par mégarde le cerf sacré qu'il avait pour compagnon, et est transformé en cyprès - l'arbre de la tristesse - par les dieux à qui il a demandé la grâce de faire couler ses larmes éternellement ${ }^{12}$.

De même, l'histoire d'Adone, qui délaisse la « Madonnina " pour les plaisirs de la chasse et est tué par un sanglier, puis changé en anémone, est une réécriture de celle d'Adonis contre qui la colère d'Artémis suscita un sanglier et dont le sang fit pousser des anémones ${ }^{13}$. Le symbolisme du sang et de la fleur est ici d'autant plus significatif que l'anémone écarlate apparaît parfois dans les représentations de la crucifixion, le rouge représentant le sang du Christ et des martyrs dans l'iconographie chrétienne.

Rolfe raconte aussi les mésaventures de Giacinto, ami de saint Michel, qui meurt en jouant au palet et dont l'âme se trouve après la mort emprisonnée dans une jacinthe à la demande de l'archange. Il s'inspire clairement ici de l'histoire de Hyacinthe, jeune prince aimé d'Apollon qui mourut frappé à la tête par un disque, et dont Apollon transforma le sang en une fleur nouvelle, la jacinthe ${ }^{14}$.

Quant à la nymphe Daphné (dont le nom signifie laurier) qui, poursuivie par Apollon, fut transformée en laurier au moment d'être atteinte par le dieu, elle devient chez Rolfe Dafne, qui refuse la protection que saint Michel lui propose ( $«$ San Michele Arcangiolo wished her well, offering to take her under his protection: but she was difficult, and nota-little silly $»^{15}$ ), et doit passer son temps de purgatoire sous la forme d'un laurier : « So she must remain until the day of judging; silent, helpless, at the mercy of the world, because in life she preferred her own will, and refused the friendship of the god with the hair of gold $»^{16}$.

Enfin, Narchiso, avatar catholicisant de Narcisse, s'éprend de sa propre image en se regardant dans les eaux d'une fontaine et, envoyé au purgatoire, se retrouve enfermé non dans un narcisse mais dans... une jonquille (" daffy-dilly », dans la langue de Toto) ${ }^{17}$.

Dans l'évocation de ce purgatoire végétal, le contexte catholique, les références au cadre eschatologique (le Jugement dernier, le paradis, l'enfer et le purgatoire, le péché et sa juste rétribution), et les allusions au "Padre Eterno", à l'« Agnello di Dio", à la " Madonnina », à « San Michele Arcangiolo » et à « Sathanas », sont en réalité secondaires par rapport aux épisodes mythologiques, qui ne sont pas véritablement "christianisés", mais plutôt transposés. La référence au purgatoire n'est qu'un prétexte qui permet à Rolfe d'écrire, après Ovide, ses propres Métamorphoses. La religion recréé par Rolfe, comme celle de beaucoup de décadents, est un catholicisme païen, où les références culturelles et littéraires à la Grèce et à Rome prennent clairement le pas sur l'eschatologie chrétienne.

\section{Sainte Rose de Lima chez l'abbé Fanfreluche}

Aubrey Beardsley, quant à lui, propose dans son roman Under the Hill (titre de la version publiée - inachevée et expurgée - de son unique roman, The Story of Venus and Tannhäuser, réécriture parodique du mythe de Tannhäuser) une pseudo-hagiographie sous forme 
d'élément dans une liste et de note en bas de page. La première moitié du chapitre IV du roman est une énumération hétéroclite des délicieuses pensées qui occupent l'abbé Fanfreluche (dont le titre est un jeu sur les initiales de Beardsley, «A. B. », dont il signait souvent ses œuvres), avatar rococo de Tannhäuser, à son réveil. Au milieu de cette rêverie matinale surgit de manière incongrue un récit de la vie de Rose de Lima, sainte péruvienne canonisée au XVII ${ }^{\mathrm{e}}$ siècle.

[He thought] [o]f Saint Rose, the well-known Peruvian virgin; how she vowed herself to perpetual virginity when she was four years old; how she was beloved by Mary, who from the pale fresco in the church of Saint Dominic, would stretch out her arms to embrace her; how she built a little oratory at the end of the garden and prayed and sang hymns in it till all the beetles, spiders, snails and creeping things came round to listen; how she promised to marry Ferdinand de Flores, and on the bridal morning perfumed herself and painted her lips, and put on her wedding frock, and decked her hair with roses, and went up a little hill not far within the walls of Lima; how she knelt there some moments calling tenderly upon Our Lady's name, and how Saint Mary descended and kissed Rose upon the forehead and carried her up swiftly into heaven. ${ }^{18}$

L'hagiographie est ici consciemment parodique. Elle est l'imitation d'un modèle qu'elle détourne de son sens initial, et met en jeu une relation à l'Église en tant qu'institution imposant des règles d'écriture, dont le caractère stéréotypé autorise le détournement. Le passage consacré à sainte Rose est inséré dans le texte entre l'image d'un lac rempli de grenouilles ( Of a great stagnant lake that must have held the subtlest frogs that ever were ») et le souvenir de la première scène de Britannicus (« He thought of the splendid opening of Racine's 'Britannicus' « $)^{19}$. Cette place vide l'hagiographie de son sens sacré. La vie de la sainte n'est plus qu'un élément dans une liste, une vision plaisante au milieu d'autres visions plaisantes. Elle perd sa signification mystique et morale et son exemplarité pour n'être plus qu'un objet esthétique, au même titre qu'une pièce de Racine, le Roman de la Rose, une peinture de Claude Le Lorrain, ou le Stabat Mater de Rossini $^{20}$, qui font aussi partie de la rêverie matinale de Tannhäuser. L'effet parodique est renforcé par la brièveté de la biographie, réduite à cinq petits tableaux censés résumer à eux seuls une vie entière d'intimité avec Dieu. Il y a quelque chose d'éminemment absurde dans la façon dont les propositions se succèdent sans transition, simplement séparées par des points-virgules. Le récit hagiographique, selon Michel de Certeau, se présente toujours comme une série de tableaux, pointant vers une transcendance qui échappe à la représentation («La manifestation [...] manque au discours qui la désigne, la fragmente et la commente en une succession de tableaux $»^{21}$ ). Beardsley reprend donc un procédé classique de l'hagiographie, mais lui fait subir une reductio ad absurdum. La fragmentation du discours n'est pas chez lui le signe d'une épiphanie irréductible à la représentation; elle n'est plus qu'une succession d'épisodes dérisoires.

Ces tableaux reprennent sur le mode parodique les topoi du genre hagiographique. Le vœu de chasteté de Rose est fait à un si jeune âge qu'il en perd son sens ; l'exil au désert et la vie érémitique deviennent retraite au fond du jardin; le don de communication avec les animaux, que l'on trouve en particulier dans les vies de saint François d'Assise, qui se faisait comprendre des loups et des oiseaux, et de saint Antoine de Padoue qui parlait aux poissons, devient capacité d'attirer les animaux les plus répugnants, tels que les scarabées, les araignées, les escargots, et autres bêtes rampantes (« creeping things »); le temps d'ascèse dans le jardin se clôt par un retour vers le monde de la cité, temps de l'illumination et du miracle, marqué ici par les préparatifs du mariage et l'enlèvement de Rose par la Vierge. 
la pâleur de la Vierge dans la fresque de l'église, les roses (dans la note qui suit ce passage, la sainte est appelée "the Flower of Lima»), sans oublier l'érotisation du rapport mystique entre la Vierge et Rose, qui se traduit dans une série de mots plus apte à décrire une relation amoureuse que la piété d'une chaste jeune fille ("beloved», "embrace", «tenderly», "kissed»). L'enlèvement de la jeune fille par la Vierge le jour de son mariage est par ailleurs le sujet d'un dessin de Beardsley qui, comme le souligne Yeats, n'est pas dépourvu d'ambiguité. W.B. Yeats, qui fut un temps lié aux milieux décadents londoniens et évoque cette époque de sa vie dans ses mémoires, décrit l'illustration en ces termes: «a St. Rose of Lima in an expensive gown decorated with roses, ascending to Heaven upon the bosom of the Madonna, her face enraptured with love, but with that form of it which is least associated with sanctity $»^{22}$.

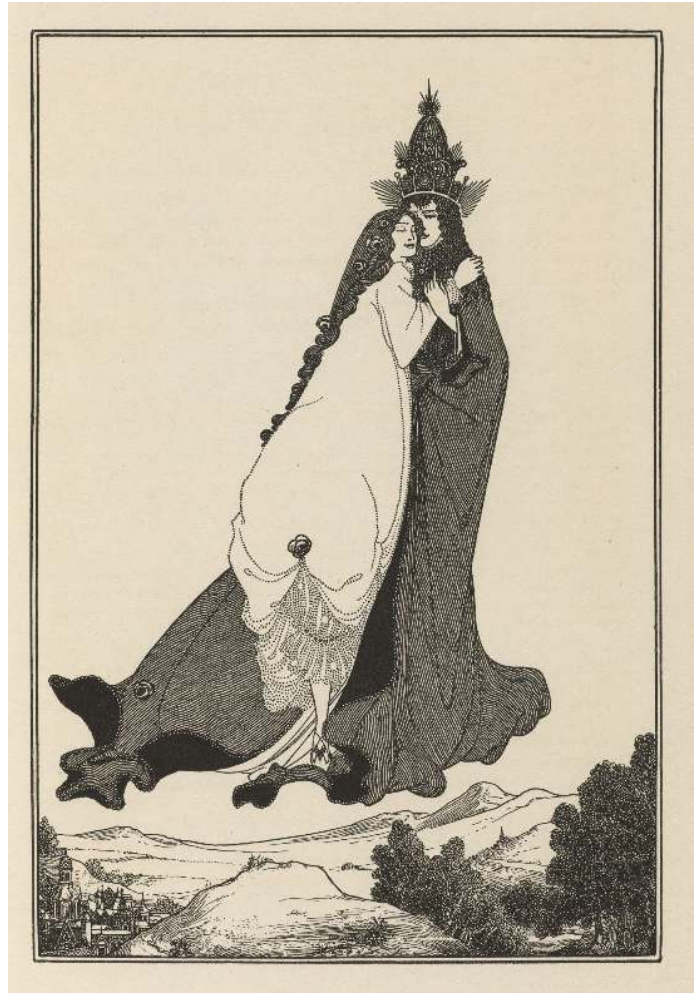

Aubrey Beardsley, «The Ascension of St. Rose of Lima », The Savoy 2 (April 1896), Houghton Library, Harvard University, Creative Commons

Comme l'hagiographie traditionnelle, qui doit ses lieux communs à la Bible, aux vies d'hommes illustres issues de l'Antiquité, et à d'autres récits hagiographiques antérieurs, comme $L a$ Légende dorée, le texte de Beardsley fait appel à une multiplicité de discours : topoi du genre hagiographique, clichés du catholicisme fin de siècle, débats contemporains, mais aussi, dans la longue note en bas de page qui accompagne le passage cité ci-dessus, textes réels ou fictifs :

All those who would respire the perfumes of Saint Rose's sanctity, and enjoy the story of the adorable intimacy that subsisted between her and Our Lady, should read Mother Ursula's 'Ineffable and Miraculous Life of the Flower of Lima,' published shortly after the canonization of Rose by Pope Clement X in 1671. 'Truly,' exclaims the famous nun, 'to chronicle the girlhood of this holy virgin makes as delicate a task as to trace the forms of some slim, sensitive plant, whose lightness, sweetness, and simplicity defy and trouble the most cunning pencil.' Mother Ursula 
certainly acquits herself of the task with wonderful delicacy and taste. A cheap reprint of the biography has lately been brought out by Chaillot and Son. ${ }^{23}$ lecteur, comme sainte Rose qui, selon la légende, reçut de Dieu le don de lecture à l'âge de quatre ans. Les auteurs de récits hagiographiques prennent toujours le soin de souligner l'authenticité des événements qu'ils rapportent. Beardsley, de même, cite ses sources, comme si la tradition textuelle dans laquelle s'inscrit son récit suffisait à en attester la valeur de vérité. Sur le mode de l'érudition parodique, il fait l'exhibition d'un prétendu savoir en nous offrant un véritable feuilletage d'hypotextes : obscurs ouvrages du XVII siècle, clichés du récit hagiographique (" respire the perfumes of Saint Rose's sanctity », «adorable intimacy», «Ineffable and Miraculous Life»), critique littéraire (« Mother Ursula certainly acquits herself of the task with wonderful delicacy and taste »), et discours publicitaire - un genre qui commence à émerger à la fin du XIX ${ }^{\mathrm{e}}$ siècle (« A cheap reprint of the biography has lately been brought out by Chaillot and Son »).

Aubrey Beardsley ne cite cependant pas sa source véritable, The Lives of St. Rose of Lima, the Blessed Colomba of Rieti and of St. Juliana Falconieri. Les Oratoriens avaient en effet publié en 1847 une série intitulée The Saints and Servants of God, essentiellement composée de traductions d'hagiographies écrites par des auteurs continentaux. La série comptait notamment une vie de Rose de Lima éditée par William F. Faber (pasteur et poète anglican, qui se convertit au catholicisme en 1845 sous l'influence de Newman), qui fut perçue comme naïve, voire superstitieuse, par beaucoup de catholiques anglais, et donna lieu à une véritable controverse. Faber était tout à fait conscient du caractère choquant que pouvait revêtir cette hagiographie pour des lecteurs anglais, puisqu'il écrit dans la préface :

English readers, who may not have been in the habit of reading the Lives of the Saints [...] may be a little startled with the LIFE OF S. ROSE. The visible intermingling of the natural and supernatural worlds [...] may offend where persons have been in the habit of paring and bating down the 'unearthly' in order to evade objections and lighten the load of the controversialist, rather than of meditating with awe and thankfulness and deep self-abasement on the wonders of God in His saints [...] and mastering the principles and general laws which are discernible even in the supernatural regions of hagiology. ${ }^{24}$

Faber défend précisément ici les caractéristiques de l'hagiographie catholique que Beardsley choisit de parodier dans Under the Hill. Le récit de Beardsley s'inspire directement de l'ouvrage préfacé par Faber, notamment des chapitres I ( Her country, her birth, her inclinations, and the vow of virginity she made at the age of five years »), VII (« Of her solitude, and the hermitage which she had built in her father's garden, that she might live quite separated from men »), XV ("Of her devotion to an image of Our Blessed Lady, to the sign of the cross, and to her dear mistress S. Catherine of Sienna ») et XX ( " Of the honour which S. Rose received after death, and of the translation of her body which took place some time afterwards »). Beardsley ne se trompe pas lorsqu'il donne 1671 comme date de publication de l'hagiographie originelle, mais contrairement à ce qu'il prétend, elle n'est pas l'œuvre de "Mother Ursula ", mais d'un certain père JeanBaptiste Feuillet ${ }^{25}$. Le texte de Beardsley offre au lecteur une mosaïque de citations réelles ou inventées, mêlant sources réelles et sources fictives, dont la juxtaposition produit un effet comique et désacralise la vie de la sainte. Comme les clercs des abbayes médiévales, à qui l'écriture hagiographique était proposée comme un exercice scolaire, Beardsley fait de son récit de la vie de sainte Rose un exercice de style, un jeu.

Polysèmes, 13 | 2015 
30 Aubrey Beardlsey et Frederick Rolfe font ainsi de l'hagiographie un récit ludique où la référence chrétienne n'est que prétexte à un véritable jeu de masques littéraire. Chez eux, la sainteté même est un texte. L'imaginaire religieux fin de siècle est un monde de l'écrit, et l'écriture décadente du catholicisme ne peut être qu'une perpétuelle réécriture. Face au spectre d'un langage qui s'épuise et se désintègre, la tradition hagiographique, mais aussi la mythologie grecque et latine chez Rolfe, la critique littéraire et le discours publicitaire chez Beardsley, offrent un vaste palimpseste, que les deux auteurs sont prompts à s'approprier sur le mode burlesque de la parodie. En tentant de revivifier une parole morte par le processus de la traduction, de la citation, de la répétition ou de la parodie, ils prennent acte du désenchantement du mot, et du monde, tout en continuant à écrire - à réécrire - en dépit de tout, tout en faisant de cette écriture - de cette réécriture - un jeu d'érudition parodique et irrévérencieux.

\section{BIBLIOGRAPHIE}

Beardsley, Aubrey. Under the Hill and Other Essays in Prose and Verse (1904). London: Paddington, 1977.

Dowling, Linda. Language and Decadence in the Victorian Fin de Siècle. Princeton: Princeton UP, 1986.

Certeau, Michel de. Article « Hagiographie ». Encyclopaedia Universalis, vol. XI, 160-164.

Faber, William Frederick (ed.). «The Life of St. Rose of Lima ». The Lives of St. Rose of Lima, the Blessed Colomba of Rieti and of St. Juliana Falconieri. London: Thomas Richardson and Son, 1847.

Pater, Walter. Marius the Epicurean (1885). London: Soho Book Company, 1995.

Rolfe, Frederick. Stories Toto Told Me (1898 et 1901). London: Collins, 1969.

Yeats, William Butler. Autobiographies (1922). London: Macmillan, 1926.

The Thirty-Nine Articles. « Articles of Religion ». The Book of Common Prayer (1662). London: Everyman's Library, 1999.

\section{NOTES}

1. Walter Pater, Marius the Epicurean, II (1885), London: Soho Book Company, 1995, 99.

2. Linda Dowling, Language and Decadence in the Victorian Fin de Siècle, Princeton: Princeton UP, 1986.

3. Le sens de l'adjectif «serafico " est transparent pour le lecteur français, «tonto " signifie « stupide » en italien, et « papagallo » perroquet.

4. Frederick Rolfe, «About the Heresy of Fra Serafico », Stories Toto Told Me, London: Collins, 1969, 206.

5. Ibid., 201-204.

6. Ibid., 205.

7. Voir Dowling, op. cit., xv. 
8. Pater, op. cit., I, 182.

9. Rolfe, op. cit., 112-113.

10. L'article XXII des Thirty-Nine Articles de 1563, un des textes fondateurs de la foi anglicane, condamne à la fois la croyance en un purgatoire et le culte des saints : "The Romish Doctrine concerning Purgatory [...], and also invocation of Saints, is a fond thing vainly invented, and grounded upon no warranty of Scripture, but rather repugnant to the Word of God ».

11. Rolfe, op. cit., 114.

12. Ibid., 114.

13. Ibid., 115.

14. Ibid., 115-117.

15. Ibid., 117.

16. Ibid.

17. Ibid., 117-118.

18. Aubrey Beardsley, Under the Hill and Other Essays in Prose and Verse, London: Paddington, 1977, 54-55.

19. Ibid., 52-53.

20. Ibid., 51-56.

21. Michel de Certeau, article « Hagiographie », Encyclopaedia Universalis, vol. XI, 160-164.

22. W.B. Yeats, Autobiographies, London: Macmillan, 1926, 411.

23. Beardsley, op. cit, 52 .

24. "The Life of St. Rose of Lima", The Lives of St. Rose of Lima, the Blessed Colomba of Rieti and of St. Juliana Falconieri, London: Thomas Richardson and Son, 1847, with an introduction by William Frederick Faber, vii.

25. «The Life of S. Rose is translated from the French of Father Jean Baptist Feuillet [...]; the copy which has been followed is the third edition, published at Paris in 1671, the year of her canonization by Clement X », Beardsley, op. cit, vi.

\section{RÉSUMÉS}

Les Décadents aiment les stratifications littéraires qui jouent aussi bien sur la tradition grécolatine que sur les codes chrétiens. Ainsi, Aubrey Beardsley et Frederick Rolfe proposent des réécritures parodiques de vies de saints, s'appropriant ainsi, dans des styles très différents, un jeu littéraire populaire chez les auteurs de la fin du XIX ${ }^{\mathrm{e}}$ siècle : dans son court roman érotique Under the Hill (1896), Beardsley propose un résumé de la vie de sainte Rose de Lima qui pastiche une hagiographie publiée en Angleterre en 1847 ; quant aux Stories Toto Told Me, contes parus dans The Yellow Book, ce sont des légendes « catholiques » racontées sur le mode mythologique, où les saints, avatars des dieux de l'olympe, batifolent entre la terre et l'au-delà. Dans ces textes, Beardsley et Rolfe reprennent sur le mode de la parodie les codes du récit hagiographique, dont le caractère stéréotypé autorise le détournement, et le vident de son sens religieux. En jouant à la fois sur la transparence et l'opacité (Beardsley nous offre un véritable feuilletage de prétendus hypotextes, tout en occultant sa source véritable qu'il reprend pourtant quasiment mot pour mot, tandis que Rolfe dissimule sous des noms de saints des personnages directement tirés des Métamorphoses d'Ovide), ils font de l'hagiographie un récit ludique où la référence chrétienne n'est que prétexte à un véritable jeu de masques littéraire. 
English Decadent writing frequently intermingles pagan and Christian - particularly Roman Catholic - references, often in a playful way. In his short erotic novel Under the Hill, Aubrey Beardsley offers a summary of Saint Rose of Lima's life, but this short narrative is in reality a pastiche of an English hagiography from 1847. In a different style, Frederick Rolfe's Stories Toto Told Me, which were originally published in The Yellow Book, are Catholic legends recast as GrecoRoman myths: in these tales, the saints are represented in the guise of olympian gods frolicking between heaven and earth. In these two parodic works, Beardsley and Rolfe play on effects of transparency and opacity to subvert the codes of hagiographic writing: Beardsley creates multiple intertextual layers, while concealing his real original source (which he nevertheless quotes verbatim), and Rolfe uses saints' names to refer to characters who are actually drawn from Ovid's Metamorphoses. The hagiographic genre is thus turned into a playful narrative, and Catholicism into a pretext for a literary game of masks.

\section{INDEX}

oeuvrecitee Stories Toto Told Me, Under the Hill, Yellow Book (The)

Mots-clés : décadence, hagiographie, intertextualité, parodie

Keywords : hagiography, intertextuality, parody

\section{AUTEURS}

\section{CLAIRE MASUREL-MURRAY}

Claire Masurel-Murray est Maître de Conférences à l'Université Paris-Sorbonne. Elle s'intéresse à la littérature victorienne, en particulier aux écrivains fin-de-siècle, ainsi qu'aux rapports entre littérature et religion. Elle a récemment publié Le Calice vide : l'imaginaire catholique dans la littérature décadente anglaise (Paris : Presses de la Sorbonne Nouvelle, 2011). 